\title{
Androgen insensitivity syndrome: a survey of diagnostic procedures and management in the UK
}

\author{
R M Viner, Y Teoh, D M Williams, M N Patterson, I A Hughes
}

\begin{abstract}
Objective-A two year survey of androgen insensitivity syndrome (AIS) to assess current diagnostic and management strategies.

Methods-Cases were ascertained by inclusion on the British Paediatric Surveillance Unit monthly report card for 24 months.

Results-Fifty one of 139 notifications were confirmed as AIS; 29 cases were complete AIS and 22 cases partial AIS. Seventy six per cent of complete AIS presented with an inguinal hernia, and half the complete AIS patients had an established family history of the disorder. Presentation in the partial AIS group was through ambiguous or undermasculinised genitalia; $59 \%$ of partial AIS were raised as male.

Conclusions-The importance of karyotyping girls with inguinal hernias is confirmed, and further attention should be given to genetic counselling for families of complete AIS patients. A large number of cases were misreported as partial AIS, emphasising the importance of undertaking a comprehensive diagnostic evaluation in intersex states. A large percentage of children with partial AIS were raised as boys despite severe genital undermasculinisation, indicating the current lack of validated measures that predict genital response to androgen treatment. The management of AIS is discussed and diagnostic guidelines provided to improve the diagnostic yield in AIS.

(Arch Dis Child 1997;77:305-309)
\end{abstract}

Keywords: androgen insensitivity syndrome; inguinal hernia; intersex

A two year national survey of the androgen insensitivity syndrome (AIS) was undertaken via the British Paediatric Surveillance Unit to determine existing diagnostic procedures and management strategies. A pilot study of patients with the partial form of AIS had shown that data on gonadal histology, anatomy of the internal genitalia, and steroid hormone concentrations were incomplete. ${ }^{1}$ AIS defines a female or ambiguous phenotype in a $46 \mathrm{XY}$ male with testes and normal testosterone production and metabolism. ${ }^{2}$ As androgens are essential for normal male sexual development and fertility, defects in androgen action are associated with abnormal sexual differentiation and fertility. ${ }^{3}$ Complete AIS is a rare $\mathrm{X}$ linked single gene disorder that results in a normal female phenotype, and typically presents in early adult life with primary amenorrhoea, although a significant number present with inguinal hernias in infancy. The significance of the relative rarity of bilateral inguinal hernias in girls is often not appreciated; it is estimated that $1-2 \%$ of such infants have complete AIS. ${ }^{4}$ The diagnosis is confirmed in both forms of AIS by a male karyotype and normal testosterone production and metabolism, in the presence of normal testicular histology, and generally the absence of müllerian duct remnants.

Partial AIS is a clinically heterogeneous disorder and presents a more complicated diagnostic problem. ${ }^{25}$ The degree of androgen unresponsiveness is variable, so the affected infant presents at birth with undermasculinised external genitalia of varying severity. Characteristically, there is micropenis with chordee, a bifid scrotum, and a perineal urethral opening, and gonads that may or may not be palpable within the bifid scrotum. The partial AIS phenotype must be differentiated from other conditions with a 46XY karyoptype, which can give rise to the same genital abnormality such as defects in testosterone biosynthesis, $5 \alpha-$ reductase deficiency, mixed gonadal dysgenesis, and true hermaphroditism. ${ }^{67}$ Accurate early diagnosis is important and has a profound bearing on the sex of rearing, genetic counselling, and subsequent management; a trial of androgen treatment in early infancy to assess the growth of penile erectile tissue may provide important information on androgen responsiveness. There is a lack of knowledge regarding the criteria used to decide the management of infants with partial AIS, particularly with respect to sex of rearing. Analysis of the cases identified through our survey suggests that guidelines for clinicians on the appropriate diagnostic and management strategies for AIS patients, particularly in the case of suspected partial AIS, would be helpful.

\section{Methods}

SURVEY PROCEDURE

Cases were ascertained by active surveillance via inclusion on the surveillance unit monthly report card for a two year period. Previous studies using multiple ascertainment methods have suggested that this reporting procedure alone provides between $60-80 \%$ case ascertainment rates. ${ }^{8}$ Cases were defined as an infant or child under 16 years of age with a 46XY karyotype and with either normal female external genitalia (defined as complete AIS) or abnormal external genitalia that are consistent 
with the partial AIS phenotype, that is, micropenis/clitoromegaly, bifid scrotum, perineoscrotal hypospadias. Clinicians were asked to report any child under their care who satisfied the case definitions, including those newly diagnosed in the past month, and those children diagnosed before the start of the study and who were still 16 years of age, or less, at the time of the study.

Once a case was notified, the referring clinician was asked to complete a questionnaire detailing further information about the clinical phenotype, family history, imaging studies of internal genitalia, gonadal histology, results of endocrine investigations, and management decisions on sex of rearing and hormone treatment. Determination of diagnosis was made from information provided in consultation with referring clinicians. If a case of AIS was confirmed as part of separate studies, referring clinicians were asked to collect blood samples from the index case and family members for DNA extraction, the venesection being coordinated with other investigations requiring ven-

Table 1 Cases notified to surveillance unit $(n=139)$

\begin{tabular}{ll}
\hline Total notifications & No \\
\hline Exclusions (n=63) & 21 \\
Over age at study & 5 \\
Duplicate notifications & 37 \\
Unconfirmed cases—no return of questionnaire & \\
Cases reported and questionnaire received (n=76) & 29 \\
Confirmed complete AIS & 22 \\
Confirmed partial AIS & 51 \\
Total AIS & \\
Other diagnoses: & 6 \\
Abnormal or dysplastic testes & 6 \\
Gonadal dysgenesis & 1 \\
Biosynthetic defect (17-hydroxydehydrogenase & \\
$\quad$ deficiency) & 1 \\
Drash syndrome & 1 \\
Hypogonadism & 1 \\
Hypopituitarism & 2 \\
Hypospadias & 2 \\
Smith-Lemli-Opitz syndrome & 1 \\
WAGR syndrome & 4 \\
Unknown diagnosis & \\
\hline
\end{tabular}

Drash=Wilms' tumour, male pseudohermaphroditism, and nephropathy.

Table 2 Mode of presentation in CAIS $(n=29)$

\begin{tabular}{lc}
\hline & No (\%) of cases \\
\hline Bilateral hernia & $13(45)$ \\
Unilateral hernia & $9(31)$ \\
Family history & $4(14)$ \\
Amniocentesis & $1(3)$ \\
Adoption karyotype & $1(3)$ \\
Unknown & $1(3)$ \\
\hline
\end{tabular}

Table 3 Details of gonad position and histology; values are number (\%)

\begin{tabular}{llll}
\hline & \multicolumn{3}{c}{ Partial AIS } \\
\cline { 4 - 4 } & $\begin{array}{l}\text { Complete AIS } \\
(n=29)\end{array}$ & $\begin{array}{l}\text { Reared male } \\
(n=13)\end{array}$ & $\begin{array}{l}\text { Reared female } \\
(=9)\end{array}$ \\
\hline Gonad position & & & \\
Bilateral intra-abdominal & $6(21)$ & $1(8)$ & $3(33)$ \\
Bilateral inguinoscrotal & $14(48)$ & $10(77)$ & $6(67)$ \\
One inguinal + one intra-abdominal & $9(31)$ & $2(15)$ & 0 \\
Unknown & 0 & 0 & 0 \\
Gonad histology & $12(41)$ & $1(8)$ & $7(78)$ \\
Bilateral normal testes & $8(28)$ & 0 & 0 \\
One normal testis & 0 & 0 & 0 \\
Abnormal & 0 & $8(62)$ & 0 \\
Not done & $9(31)$ & $4(30)$ & $2(22)$ \\
Unknown & &
\end{tabular}

^Unilaterally normal testis—other testis not biopsied or not identified. esection. Also, as part of a separate study, if a partial AIS patient was undergoing surgery to the external genitalia, clinicians were asked to provide (after parental consent) a genital skin biopsy specimen as part of the surgical procedure in order to establish fibroblast cultures. Systematic case ascertainment was confined to the surveillance unit reporting scheme. Ethical approval was granted by the Cambridge local ethics committee.

\section{Results}

Table 1 details notifications during the survey period. Fifty one of the total 139 notifications were confirmed as cases of AIS. There was insufficient information to make a diagnosis from 37 notifications. The diagnostic criteria for complete AIS and partial AIS were fulfilled in 29 and 22 cases, respectively.

\section{COMPLETE AIS}

All complete AIS patients had normal female genitalia and were reared as females. Presentation in complete AIS was predominantly by the discovery of a hernia in an apparently female infant $(76 \%)$; other cases were identified on the basis of a family history of the $\mathrm{X}$ linked disorder, or from the determination of a karyotype for other reasons (table 2). Regardless of the mode of presentation, inguinal hernia was present in $28(90 \%)$ of complete AIS patients; nine $(31 \%)$ were unilateral, $17(59 \%)$ bilateral. Table 3 shows details of gonadal position and histology. One or two palpable testes were present in approximately $80 \%$ of complete AIS patients. Gonadal histology was consistent with AIS in all patients in whom histological examination of the testes was performed (table 3). Apart from the report of a 'vestigial' uterus in one complete AIS patient, female internal genitalia were absent in all AIS patients.

\section{PARTIAL AIS}

Presentation in partial AIS was through ambiguous or undermasculinised genitalia. In $59 \%$ of the partial AIS cases, the sex of rearing was male. Factors that appeared to affect sex of rearing in partial AIS were the degree of labioscrotal fusion, site of urethral opening, whether the testes were palpable, and phallus size. All infants with unfused labia $(n=4)$ were raised as female and a further five infants with fused labia were also raised as female. All those raised as male $(n=13)$ had fused labioscrotal folds. Micropenis was reported in $54 \%$ of infants raised as males, although equal numbers were reported as having normal phallus size. Approximately $80 \%$ of partial AIS patients had one or two palpable testes. Infants with palpable testes at diagnosis were more likely to be raised male, and only one infant without palpable testes was raised as a male (table 3 ).

FAMILY HISTORY

Table 4 summarises information concerning family history. Approximately half the cases of complete AIS had an established family history of the disorder. There were four pairs of related complete AIS patients, and in four cases a history suggestive of androgen insensitivity was 
Table 4 Details of family history; values are number (\%)

\begin{tabular}{lcc}
\hline & $\begin{array}{l}\text { Complete AIS } \\
(n=29)\end{array}$ & $\begin{array}{l}\text { Partial AIS } \\
(n=22)\end{array}$ \\
\hline $\begin{array}{l}\text { Established complete AIS in } \\
\text { family }\end{array}$ & $12(41)$ & 0 \\
$\begin{array}{l}\text { Family history suggestive of AIS } \\
\text { No family history of AIS }\end{array}$ & $4(14)$ & $6(27)$ \\
Unknown & $13(45)$ & $15(68)$ \\
& 0 & $1(5)$
\end{tabular}

*This includes first and second degree female relatives with primary amenorrhoea or lack of pubic hair, and male relatives with undermasculinisation.

present. In contrast, only one quarter of partial AIS cases had a family history suggestive of AIS. Associated congenital anomalies were absent in the complete AIS group, except for one patient with red-green colour blindness. Associated anomalies were more common in the partial AIS group, with one example each of coarctation of the aorta, microphthalmos, ectodermal dysplasia, short stature with mental retardation, and chromosomal translocation 10q:16, q26.2:q21.

GONADECTOMY

Table 5 shows details of when gonadectomy was performed. This took place before puberty in half the complete AIS patients, whereas about one quarter had, or were due to have, removal of gonads after puberty. For the remainder, plans were unknown at the time of the survey. Median age for gonadectomy in complete AIS was 8 years (mean 5.5 years), with a median prepubertal gonadectomy age of 1 year (mean 1.7 years). Gonadectomy was performed in all partial AIS patients raised as females, and in none of those raised as males. Median age for gonadectomy in partial AIS patients was 21 months (mean 24.1 months).
Table 5 Timing of gonadectomy and sex of rearing

\begin{tabular}{lc}
\hline & No (\%) \\
\hline Complete AIS (timing of gonadectomy) & $14(48)$ \\
Prepubertal & $1(3)$ \\
Postpubertal & $6(21)$ \\
Planned postpubertally & $8(28)$ \\
Unknown timing & 0 \\
Not done & 0 \\
Partial AIS reared as male & $13(100)$ \\
Gonadectomy performed & 0 \\
No gonadectomy & $8(88)$ \\
Unknown & 0 \\
Partial AIS reared as female & $1(12)$ \\
Gonadectomy performed & \\
No gonadectomy & \\
Unknown & \\
\hline
\end{tabular}

Five complete AIS patients (17\%), but no partial AIS patients, raised as female were being given oestrogen replacement therapy at the time of the survey. Three partial AIS patients raised as male were receiving androgen replacement therapy. Genital surgery other than gonadectomy was performed in all the partial AIS females (vulvovaginoplasty), and in nearly half of partial AIS males; this included hypospadias repair, orchidopexy, and release of penile chordee.

\section{Discussion}

This study provides an overview of the characteristics and management of AIS in a large group of patients in the UK. The survey did not provide incidence or prevalence figures for AIS because of many unconfirmed reported cases, the limitations of case ascertainment using a single reporting method, the incomplete lifetime ascertainment of complete AIS due to the normality of the female phenotype, and confidentiality problems associated with reporting disorders of sexual development.

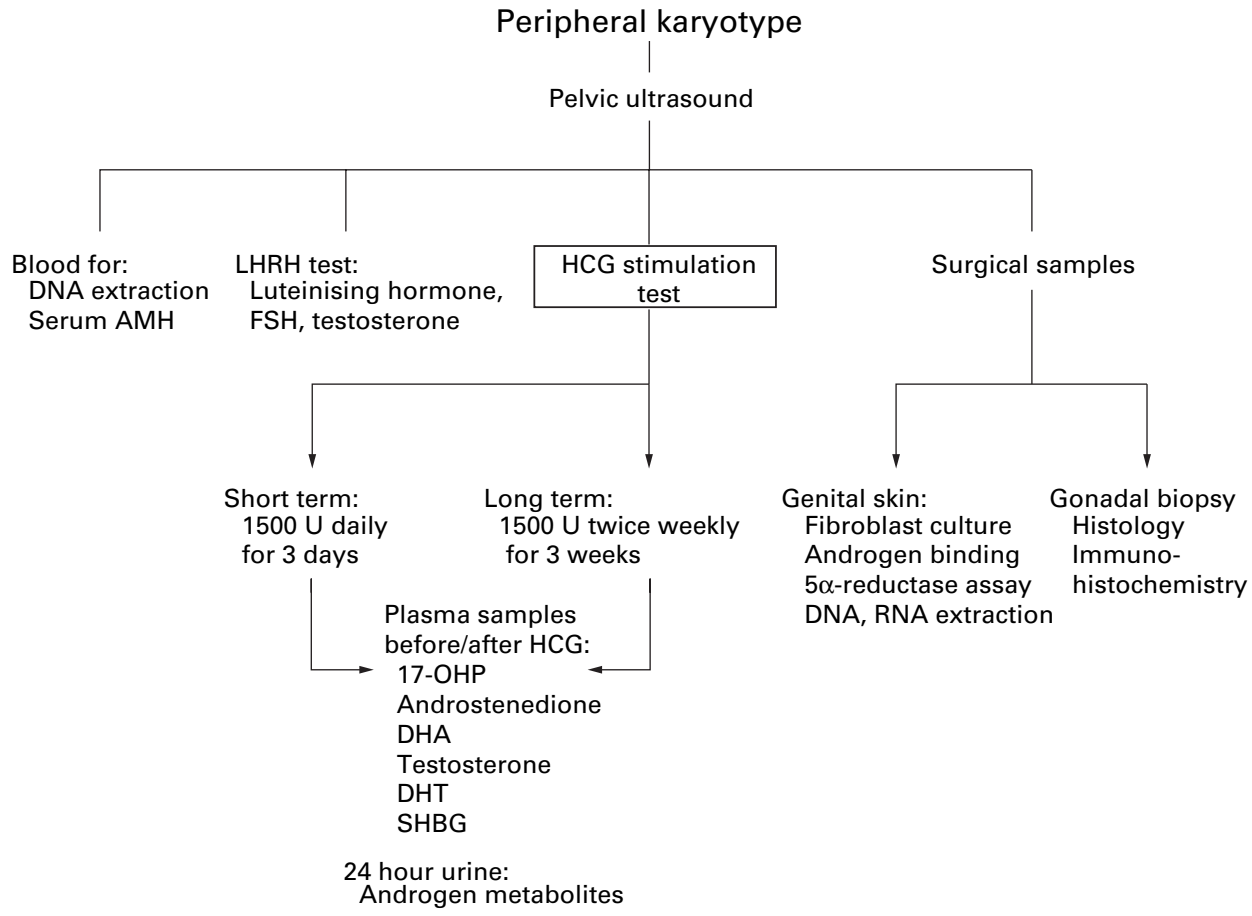

Figure 1 An investigation protocol for a patient with a partial AIS phenotype. A similar protocol can be used for complete AIS, although a human chorionic gonadotrophin test may not be necessary. AMH=antimüllerian hormone; $D H A=$ dehydroepiandrosterone; $D H T=$ dihydrotestosterone; $S H B G=$ sex hormone binding globulin. 
The presentation of three quarters of complete AIS patients through inguinal hernias, nearly half of which were unilateral, emphasises the importance of considering AIS in any female infants with hernias. Estimates of the incidence of AIS in such infants have ranged from $1-12 \%,{ }^{4}$ suggesting that any girl with an inguinal hernia should have a karyotype performed.

Nearly half the patients had a proved family history of complete AIS, yet this was the mode of presentation in only $14 \%$ of cases, suggesting that more attention be given to genetic counselling for the families of complete AIS patients. There were a number of examples when an affected sibling had previously undergone a herniorrhaphy but the underlying diagnosis had not been recognised. Karyotyping female infants with first or second degree relatives with complete AIS should be considered by clinicians. Information about the management of complete AIS is mainly limited to cases presenting before puberty as the survey was restricted to age 16 years and below. The results indicate a trend towards prepubertal gonadectomy in complete AIS, the majority of cases having surgery in infancy (median age 12 months). However, oestrogen replacement therapy had not been started in late childhood or early adolescence in several cases, raising concerns about an increased risk of osteoporosis in later life. ${ }^{10}$ The risk of malignant transformation of the gonads in adult life is well documented, ${ }^{11}$ but medical opinion remains divided about whether the testes should remain in place until after feminisation has occurred at puberty. ${ }^{2}$

Cases of partial AIS presented as expected because of abnormal genitalia at birth. Conditions misreported initially as partial AIS included testicular dysgenesis and examples of abnormal genitalia forming a component of known syndromes such as Denys-Drash, ${ }^{12}$ Smith-Lemli-Opitz, ${ }^{13}$ and Wilms' tumour, aniridia, genital anomalies, and mental retardation (WAGR). ${ }^{14}$ Other cases were associated with specific defects in androgen production. These findings emphasise that the differentiation of partial AIS from other causes of undermasculinisation requires the demonstration of normal testicular histology, adequate testosterone production after human chorionic gonadotrophin stimulation, and normal androgen metabolism. Sex assignment in partial AIS was, not surprisingly, affected by the size of the phallus and the presence of palpable testes. However, a surprisingly high percentage of partial AIS cases were raised as male even though there was severe undermasculinisation of the external genitalia. The sex hormone binding globulin response to androgens has been advocated as a simple in vivo marker of a functional defect in the androgen receptor in partial AIS patients. ${ }^{15}$ Such a marker if validated in relation to functional androgen receptor assays and clinical outcome, would be useful to guide sex of rearing decisions. The phenotypic expression of partial AIS can be so variable within affected families that the severity of undermasculinisation may be unpredictable. ${ }^{16}$

\section{Key messages}

- A karyotype is essential infants with suspected hernias

- Further attention to genetic counselling is needed for complete AIS patients to improve case ascertainment in subsequent generations

- Management of suspected partial AIS should involve a specific sequence of clinical, radiological, biochemical, and molecular investigations (fig 1 )

Establishing a diagnosis of complete AIS is straightforward with few other conditions producing a similar phenotype. However, Leydig's cell hypoplasia can be mistaken for complete AIS and may also present with ambiguous genitalia or only isolated micropenis. Loss of function mutations in the luteinising hormone receptor gene have recently been reported to cause this syndrome. ${ }^{17}$ Thus, in the absence of an increased basal testosterone, a human chorionic gonadotrophin stimulation test is indicated to verify normal androgen production in complete AIS.

The diagnosis and management of partial AIS is surrounded by inaccuracy and confusion. Many cases are interpreted as resulting from dysgenetic testes despite the lack of information on gonadal histology. Since the partial AIS phenotype may also be the expression of several other disorders, it is important to investigate cases as thoroughly as possible. Pelvic ultrasound assessment, to identify the presence or absence of müllerian structures, is particularly important as it can be done quickly, in contrast with some biochemical tests where the results may be delayed. Figure 1 illustrates a suggested flowchart for the investigation of partial AIS phenotype.

Some form of androgen insensitivity can reasonably be assumed when an XY male has normal testes as based directly on histological examination and indirectly on the absence of female internal genitalia (normal antimüllerian hormone and receptor), ${ }^{18}$ and evidence of adequate Leydig cell function based on the androgen response to human chorionic gonadotrophin stimulation. In these circumstances, it is reasonable to assume that the partial AIS phenotype may be associated with a dysfunctional androgen receptor. ${ }^{23}$ Even so, in many isolated cases of the partial AIS phenotype, androgen binding studies and mutational analysis of the androgen receptor gene are normal. ${ }^{19}$

Further androgen related genetic mechanisms involved in male sex differentiation and development remain to be defined. Detailed studies of intersex conditions can play a fundamental part in identifying genes involved in sex determination and differentiation. ${ }^{20}$ The clinical investigation of intersex patients such as those with AIS, the development of a national database of AIS patients, and continuing research into the molecular genetics of these disorders is necessary for progress in the man- 
agement of infants and children with intersex conditions.

This survey was made possible as a result of the Surveillance Unit of the Royal College of Paediatrics and Child Health (formerly the British Paediatric Association). We are grateful to unit merly the British Paediatric Association). We are grateful to unit
staff for advice and support in the organisation of the study. We staff for advice and support in the organisation of the study. We thank all paediatricians throughout the UK for their time and trouble in notifying intersex conditions to the unit and provid-
ing further information for analysis. The study was supported by ing further information for analysis. The study was supported by mation coordinator is gratefully appreciated.

1 Williams DM, Evans BAJ, Hughes IA. A clinical and biochemical analysis of 68 patients with the partial androgen insensitivity syndrome (PAIS). Horm Res 1990;3(suppl 3): 54 .

2 Patterson MN, McPhaul MJ, Hughes IA. Androgen insensitivity syndrome. Ballière's Clin Endocrinol Metab 1994;8: 379-404

3 Griffin JE. Androgen resistance-the clinical and molecular spectrum. N Engl f Med 1992;326:611-8.

4 Jagiello G, Atwell J. Prevalence of testicular feminization. Lancet 1962;i:329.

5 Quigley C, DeBellis A, Marschke K, et al. Androgen receptor defects: historical, clinical and molecular perspectives. Endocr Rev 1995;6:271-321.

6 Conte FA, Grumbach MM. Pathophysiology, genetics, nosology and diagnosis of male pseudohermaphroditism. In: Hughes IA, ed. Sex differentiation: clinical and biological In: Hughes IA, ed. Sex differentiation: clinical and biological aspects. Frontiers in

7 Berkovtiz G. Abnormalities of gonadal determination and differentiation. Semin Perinatol 1992;16:289-98.

8 British Paediatric Surveillance Unit. Eighth annual report, 1993. London: BPSU, 1994
9 Pergament E, Heimler A, Shah P. Testicular feminization and inguinal hernia. Lancet 1973;ii: $740-1$

0 VanGelderen H. Skeletal maturation in the XY female syndrome. Clin Genet 1986;30:199-201.

1 Verp $M$, Simpson J. Abnormal sexual differentiation and neoplasia. Cancer Genet Cytogenet 1987;25:191-218.

12 Mueller R. The Denys-Drash syndrome. f Med Genet 1994; 31:471-7.

13 McGaughran J, Donnai D, Clayton P. Diagnosis of Smith-Lemli-Opitz syndrome. $N$ Engl $\mathcal{F}$ Med 1994;330: 107-13.

14 Clarkson PA, Davies HR, Williams DM, et al. Mutational screening of the Wilms' tumour gene, WT1, in males with genital abnormalities. F Med Genet 1993;30:767-72.

15 Sinnecker GHG, Hiort O, Nitsche EM, et al. Functional assessment and clinical classification of androgen insensitivity in patients with mutations of the androgen receptor gene. Eur $\mathcal{F}$ Pediatr 1997;156:7-14.

16 Evans BAJ, Hughes IA, Bevan CL, MN Patterson, JW Gregory. Extreme phenotypic diversity in two siblings with the partial form of the androgen insensitivity syndrome. Arch Dis Child 1997;176:529-31.

17 Kremer H, Kraaij R, Toledo S, Post M, Fridman J, Hayashida C. Male pseudohermaphroditism due to a homozygous missense mutation of the luteinizing hormone receptor gene. Nature Genet 1995;9:160-4.

18 Rey NJR, Lordereau-Richard I, Cate R. Anti-müllerian hormone and testicular development clinical and hormonal aspects. In: Dufau M, Fabbri A, eds. Cell and molecular biology of the testis. New York: Raven Press, 1994:7-15.

19 McPhaul MJ, Marcelli M, Zoppi S, Griffin JE, Wilson JD. The spectrum of mutations in the androgen receptor gene that causes androgen resistance. 7 Clin Endocrinol Metab 1993;76:17-23.

20 Shafer AJ, Goodfellow PN. Sex determination in humans. Bioessays 1996;18:955-63. 